\title{
Potency of Asian Ginseng (Panax Ginseng) and Turmeric (Curcumin) Extract Compound in Promoting Cerebral Perfusion in Vascular Dementia: A Narrative Review
}

\author{
Velisia Putri Natalie ${ }^{1}$, Daniel Setiawan Lay ${ }^{1}$, Jennifer Luisa ${ }^{1}$, \\ Jonathan Setiawan', Ketut Wulan Ari Kartika Ardhaputri', \\ Tjokorda Gde Bagus Mahadewa ${ }^{2}$
}

${ }^{1}$ Faculty of Medicine, ${ }^{2}$ Department of Neurosurgery, Udayana University, Denpasar, Bali, Indonesia.

Corresponding Author: Velisia Putri Natalie

DOI: https://doi.org/10.52403/ijrr.20220208

\begin{abstract}
Vascular dementia $(\mathrm{VaD})$ is a neurodegenerative disease with an ever-growing number of patients in global statistics. World Health Organization (WHO) estimated that there were 50 million vascular dementia patients in 2018, with almost of $20 \%$ of it are newly diagnosed cases. North America and Europe have a similar prevalence of $\mathrm{VaD}$ patients, approximately up 15 to 20 percent of the elderly. Whilst in Asia, the number is higher, which takes up 30 percent of the elderly population. Having limited modalities in treating the condition, novel methods with considerable efficacy and safety are needed in treating dementia. Ginseng, which is the root from plants of genus Panax, contains many types of ginsenosides which have been proven to have a significant effect in alleviating cerebral hypoperfusion, the main cause of vascular dementia. Turmeric, which contains curcumin, has shown to elevate cognition and halts neurodegenerative processes, and could act as a neuroprotector through LXR-Beta/RXR signaling pathway. The combination of Panax ginseng extract and Turmeric extract is a promising new alternative drug to treat cognitive and memory problems, particularly $\mathrm{VaD}$, yet more clinical and experimental studies are required.
\end{abstract}

Keywords: Dementia, Vascular Dementia, Panax ginseng, Curcumin, Hypoperfusion

\section{INTRODUCTION}

Dementia is a clinical disease where patients experienced decreased cognition, function, and progressive changes in behavior. Cognitive deficits can be: memory loss, communication and language disorders, agnosia (inability to recognize objects), impaired reasoning function, as well as judgement and planning. According to World Health Organization (WHO), in 2018 there were around 50 million people with dementia worldwide, and nearly 10 million new cases. Vascular Dementia $(\mathrm{VaD})$ is the most common type of dementia after Alzheimer's disease (AD). Cases of vascular dementia range from $15 \%$ to $20 \%$ in North America and Europe. While in Asia the figure is $30 \%$ higher. ${ }^{[1]}$

Pathogenesis of cognitive disorders consisted of neurodegeneration and vascular degeneration, where it is closely related with hemodynamic abnormalities in the form of hypoperfusion. ${ }^{[2]}$ Cerebral blood flow maintained energy supply and metabolism in normal condition. When cerebral pressure decreased due to vascular disease, vasodilation occurs as compensatory decrease in cerebral blood flow. Once regulation reached the lower limit, cerebral blood flow gradually decreased and fraction of oxygen extraction 
increased to maintain oxygen metabolism and neuron function. ${ }^{[3]}$ Hypoperfusion is one of the early factors in the developmental process of cognitive function, where vascular oxidative stress played a major role. [2] Incidence of vascular dementia increased significantly with age.

Ginseng extract, especially panax ginseng has many positive properties, including anti-tumor, anti-inflammation, and anti-stress. When dose of ginseng extract is adjusted, it has similar effect to nimodipine, a drug commonly used for treatment of vascular dementia. [4] $\mathrm{It}$ increases cognitive aspects of learning and memory, preventing pathological changes, reduce MDA levels, and increase SOD activity and induced $\mathrm{HO}-1$ protein expression. ${ }^{[5]}$ Encapsulation is a method of using thin layer to bind a particle. The microencapsulation showed an increase in solubility of ginseng, while chemical bonding during encapsulation assists the sustained release of drug from microcapsules.

Based on the background above, this study aims to review the ability of ginseng extract with curcumin from turmeric as an anti-dementia agent via an inhibitory mechanism of cerebral hypoperfusion.

\section{METHODS}

The literature search was carried out by authors from $10^{\text {th }}$ May 2021 to $20^{\text {th }}$ September 2021 through online-based scientific journal databases, namely ScienceDirect, PubMed, and ResearchGate. The keywords used in the search were "cerebral hypoperfusion", "dementia", "vascular dementia", "neurodegenerative", "cerebrovascular", "cognitive deficit", "antidementia", "neuroprotective", "panax ginseng", "curcumin", "extraction", "encapsulation", "in vivo", "in vitro", and "clinical trial" with the use of Boolean operators such as "AND" and "OR". The inclusion criteria used in this literature review are (1) publications in the last 10 years; (2) studies in English or Indonesian. After the literature study was conducted, the study was then screened so that 127 were obtained which were appropriate as the main reference. All journals obtained were then critically examined in terms of validity, importance, and applicability. The types of data obtained are varied, but most of them are qualitative and quantitative and can be accounted for.

\section{DISCUSSION}

\section{Dementia}

Dementia is a syndrome that is usually chronic or progressive, characterized by a decline in cognitive function, and thought processing. The disease affects memory, thinking, orientation, comprehension, computation, learning capacity, language, and judgment. However, consciousness is not affected. The decline in cognitive function is usually accompanied by a decrease in emotional control, social behavior, or motivation. Dementia occurs as a result of various diseases and injuries that primarily or secondary to the brain, such as Alzheimer's disease or stroke. ${ }^{[6]}$

Presenting neuropsychiatric symptoms include apathy, agitation and depression. The patient will gradually become dependent on others to perform daily activities. Alzheimer's disease is the most common cause of dementia. The second most common cause is vascular dementia. Most dementias are progressive, irreversible, and incurable. ${ }^{[7]}$

People who have dementia before the age of 65 are said to have early dementia. The causes of dementia are not fully understood, but the result is always structural and chemical changes in the brain, leading to loss and shrinkage of nerves from brain volume. In the early stages, memory loss is associated with short-term events, and difficulty finding words. Over time, there will be greater memory loss and language difficulties. This causes difficulties in daily activities such as shopping, handling money and memorizing things. Sometimes there are other symptoms, such as anxiety and lack of 
motivation. Symptoms tend to get worse as the disease progresses until they're not capable to take care of themselves. ${ }^{[8]}$

\section{Epidemiology of Dementia}

Globally, about 50 million people suffer from dementia, $60 \%$ live in middle and low-income countries. Every year, there are nearly 10 million new cases. The total number of people with dementia is predicted to reach 82 million in 2030 and 152 million in 2050. ${ }^{[6]}$ With the percentage of vascular dementia accounting for $56 \%$ of all cases of dementia. ${ }^{[9]}$

The prevalence of dementia is increasing rapidly. Women are slightly more likely to develop dementia than men. In contrast, vascular dementia, as well as stroke and other atherosclerotic cardiovascular diseases, was more common in men. Several studies have shown that the overall prevalence of dementia varies widely between countries, influenced by cultural and socioeconomic factors. [10] Meanwhile in Southeast Asia, dementia cases is predicted doubled by 2030. The number of people living with dementia in Southeast Asia is quite large and is expected to increase, with more than 10 million people by 2030. ${ }^{[11]}$

\section{Vascular Dementia and Cerebral Hypoperfusion}

Vascular diseases have shown varying level of evidence regarding their association with cognitive impairment. There have been many studies in the recent years that suggest that vascular risk factors may contribute to the development of dementia and other neurodegeneration diseases. ${ }^{[12]}$ Due to the significant effect of vascular risk factors on cerebral hemodynamics, cerebral hypoperfusion has been suspected as a crucial link between vascular damage and dementia. [13], [14] Cerebral hypoperfusion is thought to be closely related to decreased cognitive function and other degenerative processes which may result in dementia through the process of ischemia and inflammation. [12],
[15], [16] This condition causes small-vessel diseases and negatively impact neurons that are exposed to capillary dysfunction or arteriolar disorders due to concomitant impaired vasoreactivity, blood-brain barrier (BBB) dysfunction, and inefficient extraction of oxygen and other nutrients. ${ }^{[13] \text {, }}$ [17], [18]

Low cerebral perfusion has also been associated with a higher level of impairment on the Mini Mental State Examination (MMSE). Thus, this condition is related to the severity of dementia and can predict which patients with MCI will experience worsening. ${ }^{[19], ~[20]}$ More in-depth neuropathological investigations have shown a significant decrease in myelin density of the white matter in $\mathrm{AD}$ and especially in $\mathrm{VaD}$ compared to the control group. [21], [22]

\section{Oxidative Stress}

Oxidative stress is found when the production of reactive oxygen species (ROS) is more than what the cellular antioxidant system can handle, which may cause functional disorders and cellular damage. ${ }^{18}$ In the brain parenchyme, high level of oxidative stress is closely related to the increased severity of cognitive impairment caused by cerebral hypoperfusion. ${ }^{[23]}$

Several recent studies have shown a decrease of various antioxidants in cerebral hypoperfusion conditions, including enzymatic and non-enzymatic antioxidants. ROS synthesis is associated with numerous enzymes, including nicotinamide adenine dinucleotide phosphate oxidase (Nox) which plays a major role in electron transport across membranes and superoxide formation. ${ }^{27}$ Recent studies have shown that Nox contributes greatly to oxidative stress and cognitive impairment in the hippocampus caused by cerebral hypoperfusion. ${ }^{[24]}$

Cerebral hypoperfusion has also been shown to cause activation of the brain's renin-angiotensin system (RAS). Several other studies have found that brain damage 
Velisia Putri Natalie et.al. Potency of Asian ginseng (panax ginseng) and turmeric (curcumin) extract compound in promoting cerebral perfusion in vascular dementia: a narrative review.

and cognitive decline caused by cerebral hypoperfusion can be minimized by inhibition of renin or angiotensin II type 1 (AT1) receptors. ${ }^{\text {[25], [26], [27] }}$

\section{Neuroinflammation}

Neurodegenerative diseases are associated with chronic inflammation of the central nervous system. [28], [29], [30] In vascular dementia, there is an increase of inflammatory biomarkers in plasma and cerebrospinal fluid, which is thought to indicate an inflammatory response that contributes to cognitive impairment. [24] Cerebral ischemia activates the inflammatory cascade of the immune system after the release of harmful substances, especially necrotic cell debris, which causes both repair and tissue damage. [31] In cerebral hypoperfusion, neuroinflammation of the corpus callosum and hippocampus is closely associated with white matter lesions and neuronal damage leading to impaired learning and memory. Immune cells, including microglia and astrocytes, undergo activation in which cytokines, chemokines, inflammatory pathways, and inflammatory mediators are upregulated and play an important role in the course of cerebral hypoperfusion disease. [32], [33]

Many genes associated with inflammatory response pathways such as Janus kinase (JAK)/signal transducers and activators of transcription (STAT) are coregulated with impaired axon-glial integrity in a mouse model of cerebral hypoperfusion. ${ }^{[34]}$ As a major initiator of proinflammatory cytokines in glial activation, toll-like receptor 4 (TLR4)/myeloid differential factor 88 (MyD88) signaling is stimulated in the brain alongside p38/mitogen-activated protein kinase (MAPK) downstream apoptotic signaling. ${ }^{[35]}$ In addition, nuclear factorkappaB (NF-B) and STAT3 are activated under conditions of cerebral hypoperfusion, leading to microvascular and hippocampal inflammation. ${ }^{44}$ These results suggest that inflammation and apoptosis are involved in the mechanism of hippocampus and white matter damage in chronic cerebral hypoperfusion $(\mathrm{CCH})$.

Several inflammatory mediators have also been shown to play important roles in the initiation of inflammation during cerebral hypoperfusion. Inhibition of ICAM-1 and VCAM-1 showed protection against cognitive function, suggesting that microvascular inflammatory response contributes to cerebral hypoperfusioninduced cognitive impairment. [36], [37] Another important inflammatory mediator is matrix metalloproteinase (MMP), which is one of the most potent proteinases in triggering the breakdown of the BBB in white matter lesions. Patients with vascular dementia show increased expression of MMPs in the cerebrospinal fluid, among them MMP-2 and MMP-9. ${ }^{46}$ Increased MMP-2 affects angiogenesis and selfcompensation, while inhibiting MMP-2 can reduce $\mathrm{BBB}$ disruption, glial activation, and white matter lesions. ${ }^{[24]}$ On the other hand, the resulting upregulation of MMP-9 contributes to vascular injury and demyelination. ${ }^{[38]}$ In addition, white matter lesions are accompanied by an increase in adenosine A1 receptors (A1ARs) or adenosine $\mathrm{A} 2$ receptors (A2ARs). Inhibition of both A1ARs and A2ARs exerts antiinflammatory effects and improves cognition, suggesting an important role in inflammation. [24], [39]

\section{Mitochondrial dysfunction}

Several studies have discovered that changes in energy metabolism products reflects the deficiency of energy in the brain while cerebral hypoperfusion occurs. Changes of adenosine 5'-triphosphate (ATP), adenosine 5'-diphosphate (ADP), and adenosine 5'-monophosphate (AMP), creatine phosphate, and adenosine are the metabolites that could indicate the brain is in a state of insufficient perfusion. Pyruvate dehydrogenase (PDH) as an ATP-related enzyme is also shown to be decreased, leading to less oxidative phosphorylation cycles come to pass, with the impact of 
Velisia Putri Natalie et.al. Potency of Asian ginseng (panax ginseng) and turmeric (curcumin) extract compound in promoting cerebral perfusion in vascular dementia: a narrative review.

intracellular ATP decrement. ${ }^{[24]}$ Decline of the energy levels are caused by low cerebral blood flow (CBF), that could damage the mitochondria which is the main energy source of eucaryotic cells. ${ }^{2}$ Other than producing ATPs through tricarboxylic acid cycle and oxidative phosphorylation, mitochondria is also known to participate in intracellular oxidation and reduction, as a buffer of calcium ion and its storage. Other cellular functions such as innate and adaptive immunity, activation of cytoplasmic signaling and apoptosis are also being held by the organelle. ${ }^{[40],[41],[42]}$

Mitochondrial damage caused by chronic cerebral hypoperfusion $(\mathrm{CCH})$ is also manifested in a molecular level. Excessive production of reactive oxygen species (ROS) as a result of mitochondrial dysfunction worsens the lipid peroxidase and reduction of antioxidants, SOD and GSH as examples. Intracellular reductionoxidation and free radical production imbalances causes neural damage. [43] Balance disruption of calcium ions causes reduction of mitochondrial potential membrane and respiratory complex enzymes (complex I, II, and IV) composing electron transfer chain (ETC), respiratory control index (RCI), and cytochrome $\mathrm{C}$ oxidase IV in abnormal mitochondria. [44], [45] Alteration of the oxides and ions indicated that mitochondrial function is significantly disrupted in $\mathrm{CCH} .{ }^{[46], ~[44]}$

Studies shows that apoptosis which occurs on vascular dementia plays an important role in dementia related brain injuries. ${ }^{[47]} \mathrm{Bcl}-2$ and Bax are two main proteins that regulate apoptosis with opposite mechanism. ${ }^{[48]} \mathrm{Bcl}-2$ suppresses apoptosis, yet Bax promotes it. ${ }^{[49]} \mathrm{Bcl}-2$ interferes by reducing the release of $\mathrm{C}$ cytochrome from the mitochondria that is stimulated by Bax molecules, leading to inhibition of caspase activation and decelerate apoptosis. The imbalance of the complementary proteins is also associated with neuron apopotosis, inducing neural damage and cognitive disturbances. ${ }^{[50]}$

\section{Disorder in Lipid Metabolism}

Lipid is an important content in the brain structurally and functionally. Participating in constructing the cell membrane and intracellular organelles, lipid is also involved in substance transports, neuronal signaling, oxidative stress, neural inflammation, neurodegenerative, neuroprotector, synaptogenesis, and neurogenesis, maintaining normal physiologic function of neural cells. [24], [50], [51], [52] Abnormal brain lipid metabolism concluded to reflect cerebral dysfunction.

Concentration of lipid and fatty acids are found to incline in the brain homogenate whilst in the cerebral hypoperfusion state. [24] Excessive production of free radicals which is caused by cerebral ischemia, initiated lipid peroxidase directly and indirectly. ${ }^{16}$ Over production of aldehyde and lipid peroxidase production causes cellular dysfunction through antioxidant depletion, DNA damage, and membrane dysfunction. [24], [53]

In in vivo studies involving cerebral hypoperfused mice model, pathways that are sensitive to lipid upregulation was detected to be coherently activated, respectfully mechano-gated $\mathrm{K}+$ pathway, TREK-1, TREK-2, and TRAAK. Intracellular $\mathrm{K}+$ potential was also found to alter. Lipid metabolism was also involved in early stages of cerebral hypoperfusion. In the experimented mice, unesterified fatty acid (uFA), arachidonoyl-CoA, and cytosolic phospholipase A2 (cPLA2) levels which participated in arachidonic acid metabolism were all raised. ${ }^{[54]}$

\section{Altering Growth Factor}

Growth factor (GF) is a kind of substance that encourages cell growth, proliferation, and differentiation. Among all growth factors, Brain Derived neurotrophic factor (BDNF) is the most abundant growth factor studied in cases of experimental cerebral hypoperfusion. BDNF is one of the widely distributed neurotrophic factor secreted by brain tissue and in large amounts in the CNS, especially enriched in 
the hippocampus and cortex. ${ }^{[1]}$ BDNF plays an important role in neurodevelopment, function, and survival and modulate neural plasticity and behavior to injury, and response to treatment. ${ }^{[55]}$ Decreased levels of BDNF mRNA and protein was shown in the brains of patients with Alzheimer's disease, Parkinson's disease, as well as in depression.

Several studies have revealed that BDNF is highly expressed in granule cell layer (GCL) of the dentate gyrus indicating its involvement in synaptic plasticity and neuronal development via TrkB receptors. [56] In addition, BDNF expression and release can also be altered by genetic and epigenetic variations, including histone modifications posttranslational. Histone acetylation is a highly dynamic process regulated by two class of enzymes: histone acetyltransferases (HATs) and histone deacetylases (HDACs). Histone acetylation modulated by the opposing activity of HAT and HDAC generally associated with active transcription of the gene. p300/CREBbinding protein $(\mathrm{CBP})$ is one of the three HAT families and acts as a transcriptional co-activator with HAT activity that has been shown to be important for long-term memory processing dependent on de novo gene expression. [9] Expression BDNF is also regulated by the HAT activity of p300/CBP. In addition, evidence shows that it is not only environmental manipulation that can lead to change long-term epigenetics on the BDNF promoter, but also on the treatment.

\section{Neurogliovascular Integrity Disturbance}

Not just one type of cell is responsible for pathophysiology of neurodegeneration but rather a disorder of finely tuned interactions between neuroglio-vascular unit cells (oligodendrocytes, endothelial cells, astrocytes and toe contacts, pericytes, microglia, and neurons). [10] These proangiogenic factors are important for maintaining $\mathrm{BBB}$ integrity through regulation of endothelial cells and pericytic function in angiogenesis. [57]
Damage to the neuro-glio-vascular unit impairs function $\mathrm{BBB}$, interfere with the exchange of substances between the blood and brain, and change the immune system. Efficient communication between cells within the unit neuro-glio-vascular ensures that the diameter of the cerebral blood vessels are adjusted with neuronal activity to maintain cerebral perfusion and meet metabolic demands, a process known as neurovascular coupling. ${ }^{[58]}$

Astrocytes communicate with synapses and blood vessels via end-feet processus. These processus are ideally located to mediate neurovascular coupling and facilitated by astrocytic calcium signaling and release of vasoactive substances from the end-foot terminus. [59] Astrocytic activation was detected in white matter at 7 days after BCAS surgery in mice and potentially disrupt the contact between astrocytes and blood vessel. Cerebral hypoperfusion has been shown to induce AQP4 and displacement redistribution after 3 months of BCAS. [60] Extensive gliovascular disorders can interfere with the regulation of blood flow, reducing the ability of the blood vessels brain to respond to dynamic needs in perfusion, and exacerbate hypoxia.

In humans, this condition may be worse with age-related increases in vascular rigidity and microvascular smoothing. [61] However, a direct causal relationship between disorders of the neurovascular junction and the development of white matter lesions has not been demonstrated, since the neurovascular junction disruption is related to decreased tissue metabolic requirements due to other ongoing pathological process. Displacement of astrocytic end feet or mislocalization of AQP4 from end-foot vascular contacts may further impact on glymphatic clearance pathway. [60] Until now, the impact of hypoperfusion on the glymphatic or perivascular drainage pathways have not been explored.

Pericytes are contractile cells that enclose capillary endothelial cells. These 
cells also helped regulate blood flow in the capillaries and can constrict vessels irreversibly after ischemic injury. Even though a pericyte may be an important regulators of $\mathrm{BBB}$ function, their function in hypoperfusion models for the most part is still not clearly understood. Perivascular macrophages also have not studied in hypoperfusion models, but has been shown to mediate vasculature in hypertensive rats through the production of reactive oxygen species. ${ }^{[58]}$

Basement complex membrane $(\mathrm{BM}) / \mathrm{ECM}$ is the center of maintaining neuro-glio-vascular integrity. It is an important interface between endothelial cells, mural cells and end feet astrocytes that provide important structural and functional stability through the meshwork ECM complex proteins. ${ }^{\left[{ }^{[2]}\right]}$ Mutations in proteins associated with ECM produces a form of familial small vessel disease (SVD).
Various potential mechanisms may link hypoxia with death of neural cells, many of which are associated with activation of transcription factors induced by hypoxia. Transcription factors that are hypoxia-induced can cause an increase in expression of various inflammatory cytokines which in turn lead to microglia activation, release of proinflammatory neurotoxic factors, and oxidative stress that may explain partially observed association between neuroinflammation and AD. In addition, transcription factors that are hypoxia-induced causes endothelial cells to be responsive to various proangiogenic factors, as seen in the white matter of patients with AD. Hypoxia can also result in aberrant angiogenesis and degeneration microvasculature in humans via pathways associated with advanced vascular degeneration and poor clearance of amyloid. [63]

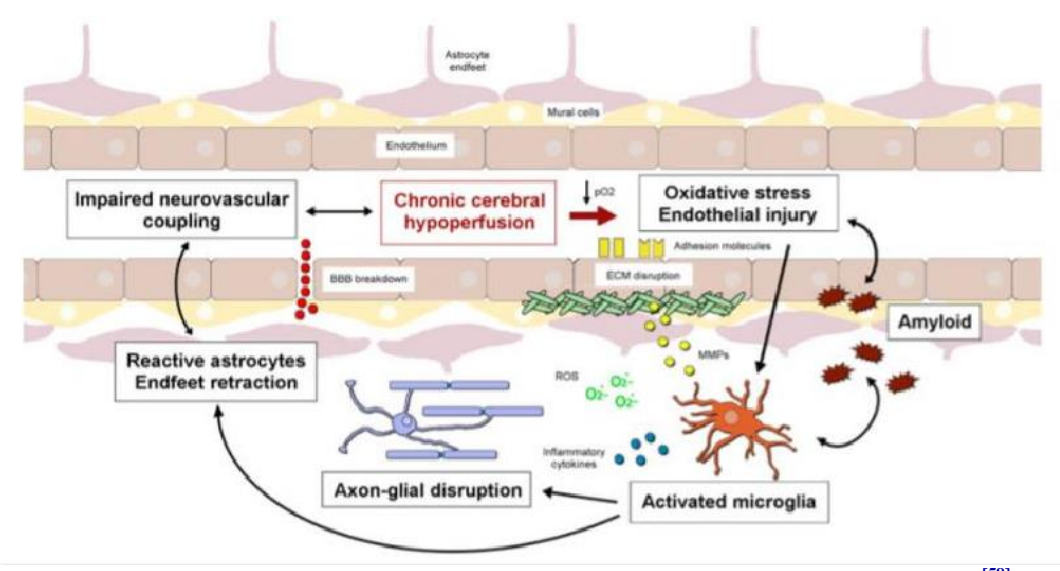

Figure 1. Overview of Hypoperfusion Pathway causing Cognitive Impairment ${ }^{[58]}$

The combination of these factors allows hypoperfusion to activate major pathways associated with hypoxia, inflammation, and $\mathrm{BBB}$ disturbances that result in progressive destruction of the neuro-glio-vascular unit (Fig. 1). Loss of integrity is likely to affect a wide variety of functions including brain-clearing pathways and regulation of $\mathrm{CBF}$ which has the potential to result in the accumulation of toxic waste products and ischemic damage. In addition, coexistent vascular risk factors such as age, CAA and hypertension can worsen neuro-glio-vascular dysfunction and its effects are being investigated recently. [58]

\section{Panax ginseng Induce Neuroprotection}

Extracts from Panax ginseng (PGE) have been taken orally as traditional medicine for thousands of years in East Asian countries such as Japan, China, and Korea. Ginseng is believed to increase vitality, prolong life, and exhibit therapeutic effects against various conditions, such as immune regulation, antitumor, antifatigue, antiaging, antioxidant, overcoming depression, diabetes, inflammation, 
dyspepsia, nervous system diseases, and many other aspects [2], [64]

Various pharmacological activities of ginseng are associated with its various active ingredients, such as ginsenosides, ginseng polysaccharides, essential oils (terpenoids, alcohols, fatty acids, etc.), peptides, and amino acids. [65], [66] PGE contains various fractions of ginseng saponins, including ginsenoside (G)-Rb1, G-Rb2，G-Rc，G-Rd，G-Re，G-Rf, G-Rg1, G-Rg2 and G-Rg3. ${ }^{[67]}$

Various modern pharmacological studies have shown that $\operatorname{Rg} 1$ can act on the nervous system in the treatment of $\mathrm{AD}$ in the elderly. One study noted that $\operatorname{Rg} 1$ ameliorated amyloid pathology, modulated amyloid precursor protein processing, and activated protein kinase/hippocampalresponding element-binding protein (PKA/CREB) signaling to induce neuroprotection. In addition, $\operatorname{Rg} 1$ also shows strong neuroprotective effect by reducing neurologic score and brain infarct volume in a mouse model of middle cerebral artery occlusion (MCAO) significantly. ${ }^{[66]}$ Research by Sun et al. (2016) showed that $\operatorname{Rg} 1$ can counteract oxidative stress and free radical injury by increasing antioxidant capacity, increasing the survival of damaged cells, reducing the amount of lactate dehydrogenase leakage and caspase-3 activation, increasing superoxide dismutase (SOD) activity and expression of heat shock protein 70 , and suppress cell apoptosis. [66], [68]

Meanwhile, Rb1 has high abundance in total ginsenosides, with excellent safety. $\mathrm{Rb} 1$ exhibits neuroprotective effects in a number of ways, including promoting neuronal growth, promoting the expression of growth-promoting kinases and helping to prevent their reduction in kinase levels, combating neurotoxins, and acting as an antiapoptotic agent. [69] Extensive studies prove that ginsenoside $\mathrm{Rd}$, one of the main active components of ginsenosides, has wide-ranging effects on the CNS, including increasing the proliferation of neural stem cells, maintaining neurogenesis after nerve injury, protection of neuronal survival, promotion of neurite growth, and protection of neurons from death in vivo and in vitro. [70], [71], [72] Many studies have shown that Rd protects neurons from I/R by reducing ROS generation and oxidative stress, stabilizing mitochondrial membrane potential and attenuating apoptotic death of hippocampal neurons after oxygen-glucose deprivation exposure. In addition, $\mathrm{Rd}$ protects against neurodegeneration caused by excess $\mathrm{Ca} 2+$, suggesting that $\mathrm{Rd}$ has a potential role in preventing and treating ischemic disease. ${ }^{[73]}$

\section{Curcumin and Its Neuroprotective Effect}

Curcumin, during the last few decades, it has been widely used in native herbal medicine for the treatment of various diseases due to its pharmacological activities including anti-inflammatory, hypoglycemic, anti-oxidant, wound healing, and others. ${ }^{[74]}$

The curcumin in dozens of recent studies has been shown to improve the effect of adverse effects caused by cerebral ischemia are precisely the effects of curcumin on the changes caused by chronic cerebral hypoperfusion. Chronic Cerebral Hypoperfusion $(\mathrm{CCH})$ is considered a high risk factor for cognitive decline in vascular dementia (VaD). Curcumin itself comes from the rhizome Curcuma Longa Linn. Many studies have reported the neuroprotective effects of curcumin in neurodegenerative diseases, including $A D$, PD and even multiple sclerosis. ${ }^{[75]}$

ABCA1 is the main protein in adipocyte membranes that plays an important role in maintaining cholesterol homeostasis by mediating high-density lipoprotein (HDL) blinding through the transport of cellular cholesterol and phospholipids to extracellular apo-A-I. It was proven in a recent study that in transgenic mice the most increased expression was ABCA1, HDL and apo-A-I. Associated with functional and structural deficiencies in neurons, ABCA1 is an important regulator of cerebral cholesterol metabolism and impaired cholesterol 
transport in the CNS. In brain cholesterol metabolism, changes in cholesterol homeostasis can cause changes in cholesterol absorption from plasma to the brain. ${ }^{[76]}$

Several studies have reported that LXR agonists can relieve brain damage caused by acute cerebral ischemia and also found that ABCA1 expression is increased. Therefore, one of the signal pathways in proving the potential of curcumin can be done through LXR/RXR-ABCAI as a new drug target for the treatment of vascular dementia. Curcumin can increase the expression of LXR-alpha and ABCA1 to promote the disposal of cholesterol in fat cells. Therefore, in our present study, we will also examine the protective role of curcumin by activating the LXR-Beta/RXR signaling pathway and modulating the target gene ABCA1 and apo-A-I expression in a mouse model of cerebral hypoperfusion. ${ }^{[76]}$

Coronary cerebral hypoperfusion induces cholesterol transport dysfunction and curcumin has great potential in causing the release of excess cholesterol by activating ABCA-1 and apoA-1 by mediating the LXR/RXR pathway thereby preventing further brain injury. In addition, curcumin is a safe and inexpensive material, easily accessible and can effectively penetrate the blood brain barrier and nerve membranes.

\section{CONCLUSION}

In the pathogenesis of cognitive disorders, nerve and vascular degeneration are closely related to hemodynamic abnormalities in the form of hypoperfusion. Vascular dementia occurs as a result of various diseases and primary injuries or affects the brain secondary. In this case, cerebral hypoperfusion is included.

Ginseng can strengthen brain function, prevent neuroinflammation and oxidative stress, and reduce or treat various neurodegenerative disorders, such as PD, $\mathrm{AD}$, traumatic brain injury, and Huntington's disease. Curcumin has a neuroprotective effect by activating the
LXR-Beta/RXR signaling pathway and modulating the target gene ABCA1 and apo-A-I expression in a mouse model of cerebral hypoperfusion. In addition, curcumin is a safe and inexpensive, easily accessible and can effectively penetrate the blood brain barrier and nerve membranes.

Collectively, these findings provide the therapeutic potential of pharmaceutical compositions containing ginseng and curcumin for the treatment of cognitive and memory disorders, particularly $\mathrm{VaD}$, in experimental and clinical studies.

\section{Acknowledgement: None}

\section{Conflict of Interest: None}

\section{Source of Funding: None}

\section{REFERENCES}

1. Wolters F, Zonneveld H, Hofman A, van der Lugt A, Koudstaal P, Vernooij M et al. Cerebral Perfusion and the Risk of Dementia. Circulation. 2017;136(8):719728.

2. Liu L, Zhang P, Li Y, Yu G. Curcumin protects brain from oxidative stress through inducing expression of UCP2 in chronic cerebral hypoperfusion aging-rats. Molecular Neurodegeneration. 2012;7(S1).

3. Nishino A, Tajima Y, Takuwa H, Masamoto $\mathrm{K}$, Taniguchi J, Wakizaka $\mathrm{H}$ et al. Longterm effects of cerebral hypoperfusion on neural density and function using misery perfusion animal model. Scientific Reports. 2016;6(1).

4. Kim M, Choi B, Lee Y, Kim D, Han Y, Jeon $\mathrm{W}$ et al. Chronic Cerebral Hypoperfusion Induces Alterations of Matrix Metalloproteinase-9 and Angiopoietin-2 Levels in the Rat Hippocampus. Experimental Neurobiology. 2018;27(4):299-308.

5. Qin W, Beck L, Zeng C, Chen Z, Li S, Zuo $\mathrm{K}$ et al. Anti-Phospholipase A2 Receptor Antibody in Membranous Nephropathy. Journal of the American Society of Nephrology. 2011;22(6):1137-1143.

6. World Health Organization, 2021.

7. Bansal N, Parle N. 2014. Dementia : An Overview. Journal of Pharmaceutical 
Velisia Putri Natalie et.al. Potency of Asian ginseng (panax ginseng) and turmeric (curcumin) extract compound in promoting cerebral perfusion in vascular dementia: a narrative review.

Technology Research and Management. Vol. 2, No. 1, pp. 29-45.

8. Dening T, Sandilyan MB (2015) Dementia: definitions and types. Nursing Standard. 29, $37,38-42$.

9. Azwar MK, Setiatk S. 2019. The Clinical Profile of Dementia in Indonesia's National General Hospital.

10. Rizzi, et al. 2014. Global Epidemiology of Dementia: Alzheimer's and Vascular Types. BioMed Research International, vol. 2014, Article ID 908915, 8 pages, 2014.

11. Poon, et al. 2018. Systematic review estimating the burden of dementia in the WHO Southeast Asia Region using Bayesian and frequentist approaches. Journal of Global Health. Vol. 10, no. 2, hh. 1-10.

12. Duncombe J, Kitamura A, Hase Y, Ihara M, Kalaria RN, Horsburgh K. Chronic cerebral hypoperfusion: A key mechanism leading to vascular cognitive impairment and dementia. Closing the translational gap between rodent models and human vascular cognitive impairment and dementia. Clinical Science. 2017.

13. De La Torre JC. Cerebral hemodynamics and vascular risk factors: Setting the stage for Alzheimer's disease. Journal of Alzheimer's Disease. 2012.

14. Drachman DA. The amyloid hypothesis, time to move on: Amyloid is the downstream result, not cause, of Alzheimer's disease. Alzheimer's Dement. 2014;

15. Akoudad S, Wolters FJ, Viswanathan A, De Bruijn RF, Van Der Lugt A, Hofman A, et al. Association of cerebral microbleeds with cognitive decline and dementia. JAMA Neurol. 2016;

16. Wolters FJ, Zonneveld HI, Hofman A, van der Lugt A, Koudstaal PJ, Vernooij MW, et al. Cerebral Perfusion and the Risk of Dementia. Circulation. 2017;

17. Zhao Z, Nelson AR, Betsholtz C, Zlokovic B V. Establishment and Dysfunction of the Blood-Brain Barrier. Cell. 2015.

18. Østergaard L, Engedal TS, Moreton F, Hansen MB, Wardlaw JM, Dalkara T, et al. Cerebral small vessel disease: Capillary pathways to stroke and cognitive decline. Journal of Cerebral Blood Flow and Metabolism. 2016.

19. Alsop DC, Dai W, Grossman M, Detre JA. Arterial spin labeling blood flow MRI: Its
Role in the early characterization of Alzheimer's disease. Journal of Alzheimer's Disease. 2010.

20. Chao LL, Buckley ST, Kornak J, Schuff N, Madison C, Yaffe K, et al. ASL perfusion MRI predicts cognitive decline and conversion from MCI to dementia. Alzheimer Dis Assoc Disord. 2010;

21. Ihara M, Polvikoski TM, Hall R, Slade JY, Perry RH, Oakley AE, et al. Quantification of myelin loss in frontal lobe white matter in vascular dementia, Alzheimer's disease, and dementia with Lewy bodies. Acta Neuropathol. 2010;

22. Barker R, Wellington D, Esiri MM, Love S. Assessing white matter ischemic damage in dementia patients by measurement of myelin proteins. J Cereb Blood Flow Metab. 2013;

23. Luca M, Luca A, Calandra C. The Role of Oxidative Damage in the Pathogenesis and Progression of Alzheimer's Disease and Vascular Dementia. Oxidative Medicine and Cellular Longevity. 2015.

24. Du SQ, Wang XR, Xiao LY, Tu JF, Zhu W, $\mathrm{He} \mathrm{T}$, et al. Molecular Mechanisms of Vascular Dementia: What Can Be Learned from Animal Models of Chronic Cerebral Hypoperfusion? Molecular Neurobiology. 2017.

25. Dong YF, Kataoka K, Toyama K, Sueta D, Koibuchi N, Yamamoto E, et al. Attenuation of brain damage and cognitive impairment by direct renin inhibition in mice with chronic cerebral hypoperfusion. Hypertension. 2011;

26. Rodriguez-Perez AI, Dominguez-Meijide A, Lanciego JL, Guerra MJ, Labandeira-Garcia JL. Dopaminergic degeneration is enhanced by chronic brain hypoperfusion and inhibited by angiotensin receptor blockage. Age (Omaha). 2013;

27. Füchtemeier M, Brinckmann MP, Foddis M, Kunz A, Po C, Curato C, et al. Vascular change and opposing effects of the angiotensin type 2 receptor in a mouse model of vascular cognitive impairment. J Perinatol. 2015;

28. Rosenberg GA, Bjerke M, Wallin A. Multimodal markers of inflammation in the subcortical ischemic vascular disease type of vascular cognitive impairment. Stroke. 2014.

29. Fakhoury M. Role of immunity and inflammation in the pathophysiology of 
Velisia Putri Natalie et.al. Potency of Asian ginseng (panax ginseng) and turmeric (curcumin) extract compound in promoting cerebral perfusion in vascular dementia: a narrative review.

neurodegenerative

diseases.

Neurodegenerative Diseases. 2015.

30. Heppner FL, Ransohoff RM, Becher B. Immune attack: The role of inflammation in Alzheimer disease. Nature Reviews Neuroscience. 2015.

31. Shichita T, Sakaguchi R, Suzuki M, Yoshimura A. Post-ischemic inflammation in the brain. Frontiers in Immunology. 2012.

32. Kim MS, Bang JH, Lee J, Han JS, Kang HW, Jeon WK. Fructus mume Ethanol Extract Prevents Inflammation and Normalizes the Septohippocampal Cholinergic System in a Rat Model of Chronic Cerebral Hypoperfusion. J Med Food. 2016;

33. Xu X, Zhang B, Lu K, Deng J, Zhao F, Zhao B qiao, et al. Prevention of Hippocampal Neuronal Damage and Cognitive Function Deficits in Vascular Dementia by Dextromethorphan. Mol Neurobiol. 2016;

34. Reimer MM, McQueen J, Searcy L, Scullion G, Zonta B, Desmazieres A, et al. Rapid disruption of axon-glial integrity in response to mild cerebral hypoperfusion. $\mathbf{J}$ Neurosci. 2011;

35. Lee KM, Bang JH, Kim BY, Lee IS, Han JS, Hwang BY, et al. Fructus mume alleviates chronic cerebral hypoperfusioninduced white matter and hippocampal damage via inhibition of inflammation and downregulation of TLR4 and p38 MAPK signaling. BMC Complement Altern Med. 2015;

36. Won JS, Kim J, Annamalai B, Shunmugavel A, Singh I, Singh AK. Protective role of snitrosoglutathione (GSNO) against cognitive impairment in rat model of chronic cerebral hypoperfusion. $\mathrm{J}$ Alzheimer's Dis. 2013;

37. Khan MB, Hoda MN, Vaibhav K, Giri S, Wang P, Waller JL, et al. Remote Ischemic Postconditioning: Harnessing Endogenous Protection in a Murine Model of Vascular Cognitive Impairment. Transl Stroke Res. 2015;

38. Seo JH, Miyamoto N, Hayakawa K, Pham LDD, Maki T, Ayata $\mathrm{C}$, et al. Oligodendrocyte precursors induce early blood-brain barrier opening after white matter injury. J Clin Invest. 2013;

39. Cheng P, Ren Y, Bai S, Wu Y, Xu Y, Pan J, et al. Chronic Cerebral Ischemia Induces Downregulation of A1 Adenosine Receptors
During White Matter Damage in Adult Mice. Cell Mol Neurobiol. 2015;

40. Jian H, Yi-Fang W, Qi L, Xiao-Song H, Gui-Yun Z. Cerebral blood flow and metabolic changes in hippocampal regions of a modified rat model with chronic cerebral hypoperfusion. Acta Neurol Belg. 2013;113(3):313-317. doi:10.1007/s13760012-0154-6

41. Van Vliet AR, Verfaillie T, Agostinis P. New functions of mitochondria associated membranes in cellular signaling. Biochim Biophys Acta - Mol Cell Res. 2014; 1843(10):2253-2262. doi:10.1016/j.bbamcr.2014.03.009

42. Weinberg SE, Sena LA, Chandel NS. Mitochondria in the regulation of innate and adaptive immunity. Immunity. 2015;42(3): 406-417. doi:10.1016/j.immuni.2015.02.002

43. Zhang X, Wu B, Nie K, Jia Y, Yu J. Effects of acupuncture on declined cerebral blood flow, impaired mitochondrial respiratory function and oxidative stress in multi-infarct dementia rats. Neurochem Int. 2014;65(1): 23-29. doi:10.1016/j.neuint.2013.12.004

44. Du J, Ma M, Zhao Q, et al. Mitochondrial bioenergetic deficits in the hippocampi of rats with chronic ischemia-induced vascular dementia. Neuroscience. 2013;231:345-352. doi:10.1016/j.neuroscience.2012.11.062

45. Wang XR, Shi GX, Yang JW, et al. Acupuncture ameliorates cognitive impairment and hippocampus neuronal loss in experimental vascular dementia through Nrf2-mediated antioxidant response. Free Radic Biol Med. 2015;89:1077-1084. doi:10.1016/j.freeradbiomed.2015.10.426

46. Zhang X, Wu B, Nie K, Jia Y, Yu J. Effects of acupuncture on declined cerebral blood flow, impaired mitochondrial respiratory function and oxidative stress in multi-infarct dementia rats. Neurochem Int. 2014;65(1): 23-29. doi:10.1016/j.neuint.2013.12.004

47. Liu Z, Hu M, Lu P, et al. Cerebrolysin alleviates cognitive deficits induced by chronic cerebral hypoperfusion by increasing the levels of plasticity-related proteins and decreasing the levels of apoptosis-related proteins in the rat hippocampus. Neurosci Lett. 2017;651:7278. doi:10.1016/j.neulet.2017.04.022

48. Min JJ, Huo XL, Xiang LY, et al. Protective effect of Dl-3n-butylphthalide on learning and memory impairment induced by chronic 
Velisia Putri Natalie et.al. Potency of Asian ginseng (panax ginseng) and turmeric (curcumin) extract compound in promoting cerebral perfusion in vascular dementia: a narrative review.

intermittent hypoxia-hypercapnia exposure. Sci Rep. 2014;4:1-9. doi:10.1038/srep05555

49. Hwang L, Choi IY, Kim SE, et al. Dexmedetomidine ameliorates intracerebral hemorrhage-induced memory impairment by inhibiting apoptosis and enhancing brainderived neurotrophic factor expression in the rat hippocampus. Int J Mol Med. 2013; 31(5):1047-1056.

doi:10.3892/ijmm.2013.1301

50. Wang Q, Yan J, Chen X, et al. Statins: Multiple neuroprotective mechanisms in neurodegenerative diseases. Exp Neurol. 2011;230(1):27-34. doi:10.1016/j.expneurol.2010.04.006

51. Kang J, Rivest S. Lipid metabolism and neuroinflammation in alzheimer's disease: A role for liver X receptors. Endocr Rev. 2012;33(5):715-746. doi:10.1210/er.20111049

52. Giannopoulos PF, Joshi YB, Praticò D. Novel lipid signaling pathways in Alzheimer's disease pathogenesis. Biochem Pharmacol. 2014;88(4):560-564. doi:10.1016/j.bcp.2013.11.005

53. Miura T. The peroxidase activity of ADM$\mathrm{Fe} 3+$ cooperates with lipid peroxidation: The participation of hydroperoxide and hydroxyl radicals in the damage to proteins and DNA. Chem Biol Interact. 2015;236: 67-73. doi:10.1016/j.cbi.2015.04.019

54. Bhattacharjee AK, White L, Chang L, et al. Bilateral common carotid artery ligation transiently changes brain lipid metabolism in rats. Neurochem Res. 2012;37(7):14901498. doi:10.1007/s11064-012-0740-2

55. Park H, Poo M. Neurotrophin regulation of neural circuit development and function. Nature Reviews Neuroscience. 2012;14(1): 7-23.

56. Koppel I, Timmusk T. Differential regulation of Bdnf expression in cortical neurons by class-selective histone deacetylase inhibitors. Neuropharmacology. 2013 Dec 1;75:106-15.

57. Shindo A, Tanemura H, Yata K, Hamada K, Shibata M, Umeda Y, Asakura F, Toma N, Sakaida H, Fujisawa T, Taki W. Inflammatory biomarkers in atherosclerosis: pentraxin 3 can become a novel marker of plaque vulnerability. PloS one. 2014 Jun 17;9(6):e100045.

58. Duncombe J, Kitamura A, Hase Y, Ihara M, Kalaria RN, Horsburgh K. Chronic cerebral hypoperfusion: a key mechanism leading to vascular cognitive impairment and dementia. Closing the translational gap between rodent models and human vascular cognitive impairment and dementia. Clinical Science. 2017 Sep 28;131(19):2451-68.

59. Bazargani N, Attwell D. Astrocyte calcium signaling: the third wave. Nature neuroscience. $2016 \mathrm{Feb}$;19(2):182-9.

60. Iliff JJ, Wang M, Liao Y, Plogg BA, Peng W, Gundersen GA, Benveniste H, Vates GE, Deane R, Goldman SA, Nagelhus EA. A paravascular pathway facilitates CSF flow through the brain parenchyma and the clearance of interstitial solutes, including amyloid $\beta$. Science translational medicine. 2012 Aug 15;4(147):147ra111-.

61. Brown WR, Thore CR. Cerebral microvascular pathology in ageing and neurodegeneration. Neuropathology and applied neurobiology. 2011 Feb;37(1):5674.

62. Joutel A, Haddad I, Ratelade J, Nelson MT. Perturbations of the cerebrovascular matrisome: a convergent mechanism in small vessel disease of the brain?. Journal of Cerebral Blood Flow \& Metabolism. 2016 Jan;36(1):143-57.

63. Yata K, Tomimoto H. Chronic cerebral hypoperfusion and dementia. Neurology and Clinical Neuroscience. 2014 Sep;2(5):12934.

64. Yata K, Tomimoto H. Chronic cerebral hypoperfusion and dementia. Neurology and Clinical Neuroscience. 2014 Sep;2(5):12934.

65. Yang Y, Du K, Liu Z, Lu X. Endothelial nitric oxide synthase (eNOS) 4b/a gene polymorphisms and coronary artery disease: evidence from a meta-analysis. Int $\mathrm{J}$ Mol Sci. 2014;15(5):7987-8003.

66. Peña, I. Dela et al. (2014) 'Effects of ginseol k-g3, an rg3-enriched fraction, on scopolamine-induced memory impairment and learning deficit in mice', Journal of Ginseng Research.

67. Huang, L. et al. (2014) 'Long-term inhibition of Rho-kinase restores the LTP impaired in chronic forebrain ischemia rats by regulating GABAA and GABAB receptors', Neuroscience

68. Ban, J. Y. et al. (2012) 'Korean red ginseng protects against neuronal damage induced by transient focal ischemia in rats', Experimental and Therapeutic Medicine 
Velisia Putri Natalie et.al. Potency of Asian ginseng (panax ginseng) and turmeric (curcumin) extract compound in promoting cerebral perfusion in vascular dementia: a narrative review.

69. Sun, Z. G. et al. (2016) 'Protective effects of ginsenoside $\operatorname{Rg} 1$ against hydrogen peroxideinduced injury in human neuroblastoma cells', Neural Regeneration Research.

70. Wang, H. C. et al. (2012) 'Protective effect of ginsenoside $\mathrm{Rb} 1$ on hippocampal neuron injury after treated by $A \beta$-amyloid protein in rats', Journal of Jilin University Medicine Edition.

71. Lin, T. et al. (2012) 'Promotive effect of ginsenoside $\mathrm{Rd}$ on proliferation of neural stem cells in vivo and in vitro', Journal of Ethnopharmacology

72. Wang, B. et al. (2013) 'Ginsenoside Rd maintains adult neural stem cell proliferation during lead-impaired neurogenesis', Neurological Sciences.

73. Ong, W. Y. et al. (2015) 'Protective effects of ginseng on neurological disorders', Frontiers in Aging Neuroscience

74. Wan, Q. et al. (2017) 'Ginsenoside Reduces Cognitive Impairment During Chronic Cerebral Hypoperfusion Through BrainDerived Neurotrophic Factor Regulated by Epigenetic Modulation', Molecular Neurobiology.

75. Lin, T. et al. (2012) 'Promotive effect of ginsenoside $\mathrm{Rd}$ on proliferation of neural stem cells in vivo and in vitro', Journal of Ethnopharmacology.

76. de Alcântara, G. F., Simões-Neto, E., da Cruz, G. M., Nobre, M. E., Neves, K. R., de Andrade, G. M., Brito, G. A., \& Viana, G. S. (2016). Curcumin reverses neurochemical, histological and immunohistochemical alterations in the model of global brain ischemia. Journal of traditional and complementary medicine, 7(1), 14-23. https://doi.org/10.1016/j.jtcme.2015.10.001

77. Rocha-Ferreira, E., Sisa, C., Bright, S., Fautz, T., Harris, M., Contreras Riquelme, I., Agwu, C., Kurulday, T., Mistry, B., Hill, D., Lange, S., \& Hristova, M. (2019). Curcumin: Novel Treatment in Neonatal Hypoxic-Ischemic Brain Injury. Frontiers in physiology, 10, 1351. https://doi.org/10.3389/fphys.2019.01351

How to cite this article: Velisia Putri Natalie, Daniel Setiawan Lay, Jennifer Luisa et.al. Potency of Asian ginseng (panax ginseng) and turmeric (curcumin) extract compound in promoting cerebral perfusion in vascular dementia: a narrative review. International Journal of Research and Review. 2022; 9(2): 40-52. DOI: https://doi.org/10.52403/ijrr. 20220208 